\title{
Juxta-adrenal malignant schwannoma with lymph node metastases
}

\author{
Yu Seob Shin, MD; Hyung Jin Kim, MD; Myung Ki Kim, MD
}

Department of Urology, Chonbuk National University Medical School, and Research Institute of Clinical Medicine of Chonbuk National University-Biomedical Research Institute of Chonbuk National University Hospital, Jeoniu, South Korea

Cite as: Can Urol Assoc J 2013;7(9-10):e657-9. http://dx.doi.org/10.5489/cuaj.1217 Published online October $9,2013$.

\section{Abstract}

Juxta-adrenal malignant schwannoma is a very rare tumour of neural crest cell origin. A 69-year-old woman visited for proper management of incidentally detected adrenal mass as part of a dyspepsia workup. Metabolic evaluation was unremarkable and imaging results suggested a malignant adrenal mass with lymph node metastases. The patient underwent a left adrenalectomy with para-aortic lymphadenectomy. The diagnosis and its origin was confirmed by histologic and immunohistochemical studies. We report the first case of juxta-adrenal malignant schwannoma with lymph node metastases, and review the literature on this rare tumour.

\section{Introduction}

Schwannomas are rare tumours, usually benign, originating from the Schwann sheath of the peripheral or cranial nerves. Visceral schwannomas are rare and only a few cases of adrenal schwannomas have been reported..$^{1-3}$ More particularly, juxta-adrenal malignant schwannoma are extremely rare and lymph node metastasis is uncommon. ${ }^{4}$ We report the first case of juxta-adrenal malignant schwannoma with lymph node metastases.

\section{Case report}

A 69-year-old female was referred to our institution for treatment of incidentally detected left adrenal as a part of a dyspepsia workup. History and physical examination were unremarkable. Abdominal magnetic resonance imaging (MRI) with intravenous administration of gadolinium revealed a $6.5 \times 6.6-\mathrm{cm}$ lobular mass arising from the medial limb of the left adrenal gland. The mass demonstrated early peripheral enhancement with no significant washout (Fig. 1, part a). Multiple para-aortic lymphadenopathies were present (Fig. 1, part b). Metabolic workup, including serum electrolytes, cortisol, urinary metanephrine and vanillylmandelic acid (VMA), were within normal ranges. The patient then underwent a left adrenalectomy and lymph node dissection through transperitoneal approach. The tumour was well-encapsulated without invasion of kidney. There was no grossly normal adrenal gland and a few enlarged paraaortic lymph nodes were identified. The postoperative course was uneventful. Pathologic evaluation revealed a solitary tumour measuring $7.5 \times 5.0 \times 5.0 \mathrm{~cm}$ in overall dimensions with $111 \mathrm{~g}$ weight (Fig. 2, part a). Histologically, the tumour exhibited characteristics of a malignant nerve sheath neoplasm that appeared to arise from the juxta-adrenal gland (Fig. 2, part b) with para-aortic lymph node metastasis (Fig. 2, part c). Immunohistochemical studies revealed that cells were uniformly S-100 positive (Fig. 3, part a), vimentin positive (Fig. 3, part b), and were negative for smooth muscle actin (Fig. 3, part c). These findings were consistent with malignant juxta-adrenal schwannoma. Computed tomography $(\mathrm{CT})$ of the abdomen after 3 months showed a small liver metastasis and others were unremarkable. The patient underwent percutaneous radiofrequency ablation for hepatic metastasis.

\section{Discussion}

Adrenal schwannomas are exceedingly rare. Only 20 cases have been reported and most of them preoperatively diagnosed as non-secreting adrenal masses. Their origin appears to be in the Schwann cells of the nerve fibers innervating the adrenal medulla. Adrenal schwannoma is a usually benign, slow-growing, encapsulated neoplasm in which the principal component arises from neural crest cells and comprises differentiated Schwann cells in a poorly-collagenized stroma. An extensive review revealed only 2 cases of malignant juxta-adrenal schwannoma. ${ }^{4,5}$ Considering the rich neural supply of the adrenal gland, a juxta-adrenal malignant 


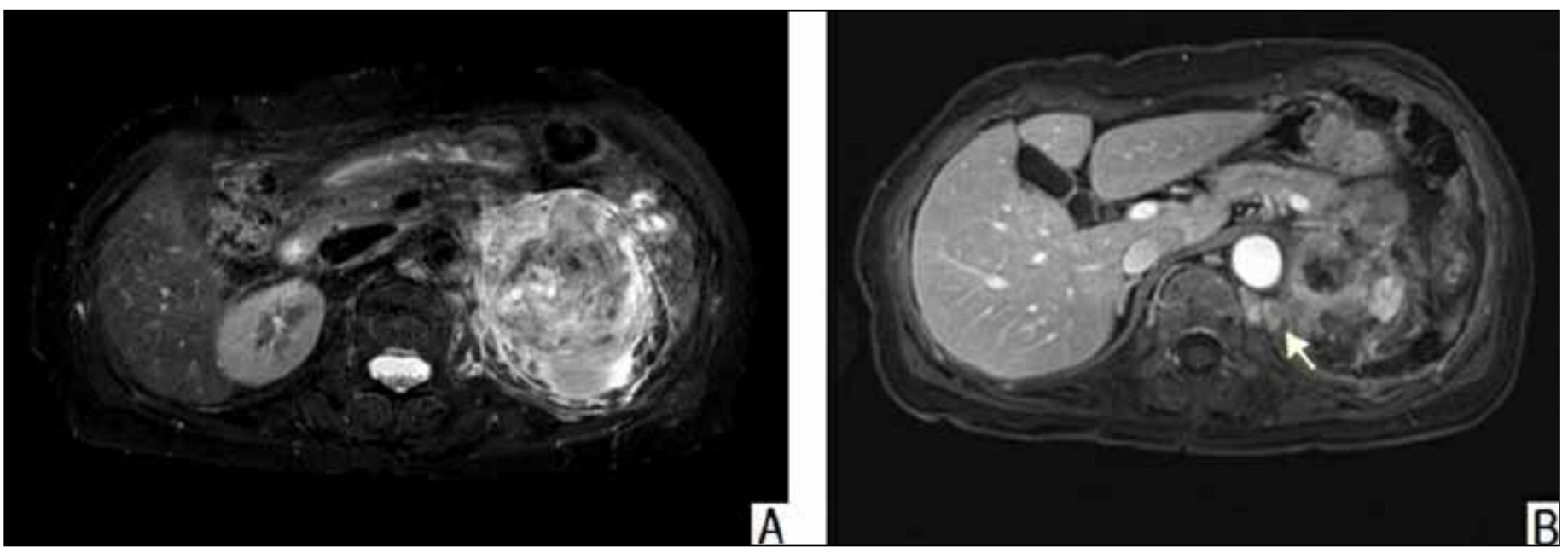

Fig. 1. A. T2-weighted magnetic resonance image of the abdomen after intravenous gadolinium was administered showing a large mass in left adrenal gland. B. Image findings of para-aortic lymph node metastases (arrow).

schwannoma is plausible. ${ }^{4}$ The malignant form is frequently associated with von Recklinghausen syndrome (4\% of cases) or other types of neurofibromatosis. ${ }^{6}$

Adrenal schwannomas are typically found incidentally. However, patients may present with clinical symptoms secondary to the mass effect of the tumour. Given the nonfunctional nature of schwannomas, only positive hormonal studies, such as elevated urine metanephrines in most cases of pheochromocytoma, can unequivocally rule out the diagnosis of schwannoma. On CT, a schwannoma appears as a well-demarcated, round or oval mass that may be homogeneous, as in the present case. However, other cases have shown prominent cystic degeneration and calcifications. With the addition of contrast, schwannomas may demonstrate variable homogeneous or heterogeneous enhancement. ${ }^{6}$ On T1-weighted MRI, a schwannoma typically presents a high- or iso-signal intensity, whereas T2-weighted images are usually low-signal intensity lesion. ${ }^{5}$

Histomorphological examination can provide a definitive diagnosis, demonstrating neoplastic cells that simulate the appearance of differentiated Schwann cells that are wellcircumscribed and composed of spindle cells organized as cellular areas with nuclear palisading (Antoni A) and paucicellular areas (Antoni B). ${ }^{7}$ Immunohistochemical staining can provide secondary confirmation of histology with diffusely positive staining for S-100 protein and vimentin. A small subset of schwannomas may be indistinguishable from neurofibromas, due to their similar histologic appearance and positive staining for S-100 protein. Fine and colleagues ${ }^{8}$ demonstrated that, in such instances, a positive stain for calretinin, a calcium-binding protein belonging to the same protein family as S-100 that is expressed in schwannoma but not neurofibroma, allows discrimination of these two entities.

Surgical treatment offers the best possibility of cure or long-term palliation. Despite the relatively slow growth rate, a high percentage of patients present with local recurrence and lung, liver or bone metastasis, even after radical surgery. Results of treatment with radiation and chemotherapy have also been disappointing. ${ }^{4}$ Lymph node metastases are

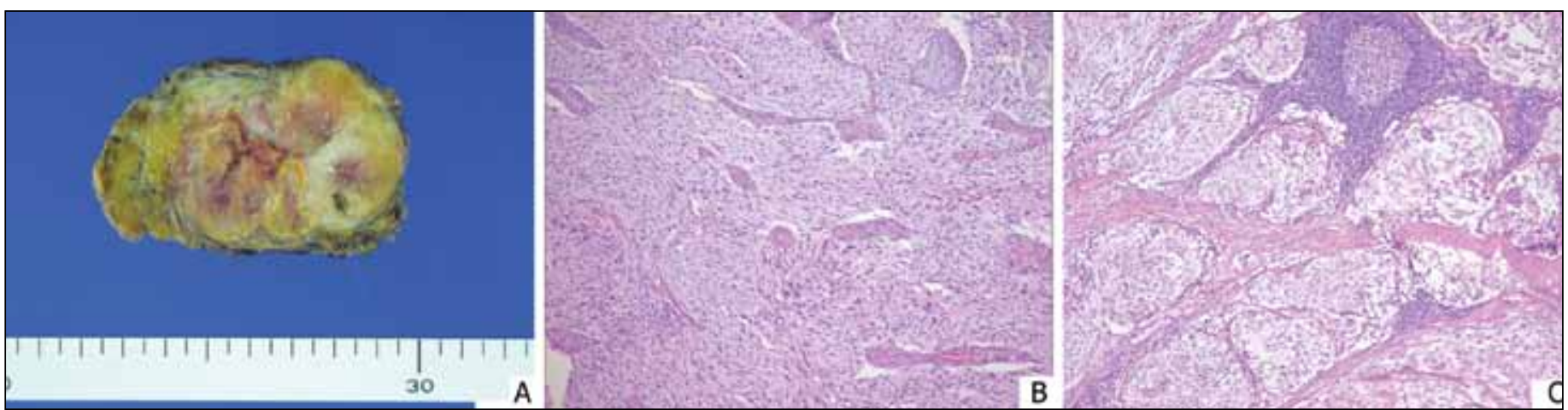

Fig. 2. A. Gross findings of juxta-adrenal mass. The tumours were yellowish-white colored and $7.5 \times 5.0 \times 5.0 \mathrm{~cm}$ in overall dimensions with $111 \mathrm{~g}$ weight. $B$. Histologically, the tumour was composed of spindle cells arranged in sweeping fascicles. The spindle cells showed nodular or whorled appearance with perivascular accentuation (hematoxylin and eosin stain; original magnification $\times 200$ ). C. Microscopic findings of lymph node metastasis. 

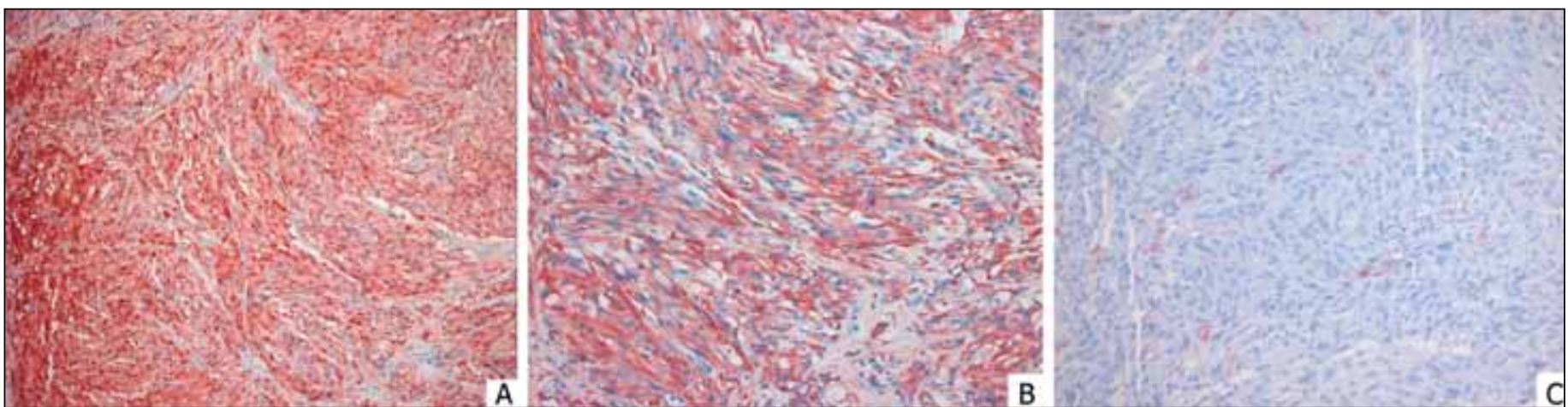

Fig. 3. A. Immunohistochemical studies showed positive reaction of the tumour cells for S-100 protein confirming their neural differentiation (S-100 protein; original magnification $\times 200$ ). B. Positive reaction of the tumour cells for vimentin (vimentin; original magnification $\times 200$ ). C. Negative reaction of the tumour cells for SMA (SMA; original magnification $\times 200$ ).

uncommon. Hence, lymphadenectomy rarely plays a role in tumour management, ${ }^{4}$ but the present case was shown to be lymph node metastases.

\section{Conclusion}

We report the first case of an incidentally discovered malignant schwannoma arising from the juxta-adrenal gland with lymph node metastases. Given the rarity of this tumour and lack of definitive nonhistologic diagnostic modalities, juxtaadrenal schwannoma remains a diagnosis of exclusion that nonetheless deserves a place on the differential of an incidentally discovered adrenal mass.

Competing interests: None declared.

This paper has been peer-reviewed.

\section{References}

1. Arena V, De Giorgio F, Drapeau CM, et al. Adrenal Schwannoma. Report of two cases. Folia Neuropathol 2004;42:177-9.

2. Bedard YC, Horvath E, Kovacs K. Adrenal schwannoma with apparent uptake of immunoglobulins. Ultrastruct Pathol 1986; 10:505-13. http://dx.doi.org/10.3109/01913128609007208

3. Lau SK, Spagnolo DV, Weiss LM. Schwannoma of the adrenal gland: report of two cases. Am I Surg Pathol 2006;30:630-4. http://dx.doi.org/10.1097/01.pas.0000194739.80174.26

4. Fabbro MA, Costa L, D'Agostino S, et al. Juxta-adrenal malignant schwannoma. Pediatr Surg Int 1997;12:532-4. http://dx.doi.org/10.1007/BF01258720

5. Jow W, Satchidanand S, Spinazze E, et al. Malignant juxtadrenal schwannoma. Urology 1991;38:383-6. http://dx.doi.org/10.1016/0090-4295(91)80160-9

6. Garcia G, Anfossi E, Prost J, et al. Benign retroperitoneal schwannoma: report of three cases. Prog Urol 2002;12:450-3.

7. Korets R, Berkenblit R, Ghavamian R. Incidentally discovered adrenal schwannoma. JSLS 2007;11:113-5.

8. Fine SW, MCClain SA, Li M. Immunohistochemical staining for calretinin is useful for differentiating schwannomas from neurofibromas. Am J Clin Pathol 2004;122:552-9. http://dx.doi.org/10.1309/ AGBGTBRJ4WOBC7LN

Correspondence: Dr. Myung Ki Kim, Department of Urology, Medical School, Chonbuk National University, 634-18, Geumam-dong, Deokjin-gu, Jeonju 561-712, Republic of Korea; fax: +82-63250-1564; mkkim@jbnu.ac.kr 